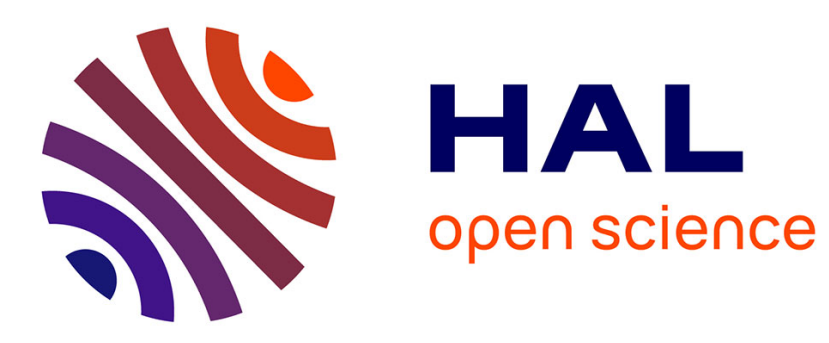

\title{
Saccadic lateropulsion in Wallenberg syndrome: a window to access cerebellar control of saccades?
}

Caroline Tilikete, Ansgar Koene, Norbert Nighoghossian, Alain Vighetto, Denis Pelisson

\section{- To cite this version:}

Caroline Tilikete, Ansgar Koene, Norbert Nighoghossian, Alain Vighetto, Denis Pelisson. Saccadic lateropulsion in Wallenberg syndrome: a window to access cerebellar control of saccades?. Experimental Brain Research, 2006, 174 (3), pp.555-565. 10.1007/s00221-006-0495-6 . hal-02196779

\section{HAL Id: hal-02196779 \\ https://hal.science/hal-02196779}

Submitted on 29 Jul 2019

HAL is a multi-disciplinary open access archive for the deposit and dissemination of scientific research documents, whether they are published or not. The documents may come from teaching and research institutions in France or abroad, or from public or private research centers.
L'archive ouverte pluridisciplinaire HAL, est destinée au dépôt et à la diffusion de documents scientifiques de niveau recherche, publiés ou non, émanant des établissements d'enseignement et de recherche français ou étrangers, des laboratoires publics ou privés. 
archives-ouvertes

\section{Saccadic lateropulsion in Wallenberg syndrome: a window to access cerebellar control of saccades?}

Caroline Tilikete, Ansgar Koene, Norbert Nighoghossian, Alain Vighetto, Denis Pelisson

\section{To cite this version:}

Caroline Tilikete, Ansgar Koene, Norbert Nighoghossian, Alain Vighetto, Denis Pelisson. Saccadic lateropulsion in Wallenberg syndrome: a window to access cerebellar control of saccades?. Experimental Brain Research, Springer Verlag, 2006, 174 (3), pp.555-565. 10.1007/s00221-006-0495-6 . hal-02196779

\section{HAL Id: hal-02196779}

https://hal.archives-ouvertes.fr/hal-02196779

Submitted on 29 Jul 2019

HAL is a multi-disciplinary open access archive for the deposit and dissemination of scientific research documents, whether they are published or not. The documents may come from teaching and research institutions in France or abroad, or from public or private research centers.
L'archive ouverte pluridisciplinaire HAL, est destinée au dépôt et à la diffusion de documents scientifiques de niveau recherche, publiés ou non, émanant des établissements d'enseignement et de recherche français ou étrangers, des laboratoires publics ou privés. 
Caroline Tilikete $\cdot$ Ansgar Koene $\cdot$ Norbert Nighoghossian

Alain Vighetto $\cdot$ Denis Pélisson

\section{Saccadic lateropulsion in Wallenberg syndrome: a window to access cerebellar control of saccades?}

\begin{abstract}
Saccadic lateropulsion is characterized by an undershoot of contralaterally directed saccades, an overshoot of ipsilaterally directed saccades and an ipsilateral deviation of vertical saccades. In Wallenberg syndrome, it is thought to result from altered signals in the olivocerebellar pathway to the oculomotor cerebellar network. In the current study we aimed to determine whether saccadic lateropulsion results from a cerebellar impairment of motor related signals or visuo-spatial related signals. We studied the trajectory, the accuracy, the direction and the amplitude of a variety of vertical and oblique saccades produced by five patients and nine control subjects. Some results are consistent with previous data suggesting altered motor related signals. Indeed, the horizontal error of contralesional saccades in patients increased with the desired horizontal saccade size. Furthermore, the initial directional error measured during the saccadic acceleration phase was smaller than the global directional error, suggesting that the eye trajectory curved progressively. However, some other results suggest that the processes that specify the horizontal spatial goal of the saccades might be impaired in
\end{abstract}

C. Tilikete $\cdot$ A. Vighetto

Neuro-Ophthalmology Department, Université Claude

Bernard Lyon-I, Hospices Civils de Lyon,

59 Bd Pinel, 69677 Bron Cedex, France

N. Nighoghossian

Stroke Department, Université Claude Bernard Lyon-I,

Hospices Civils de Lyon, 59 Bd Pinel,

69677 Bron Cedex, France

C. Tilikete $(\varangle) \cdot$ A. Koene $\cdot$ A. Vighetto $\cdot$ D. Pélisson Espace et Action, Unité 534, INSERM/Université Claude Bernard Lyon-I, 16 avenue du doyen Lépine, 69676 Bron Cedex, France E-mail: tilikete@lyon.inserm.fr

Tel.: +33-4-72913428

Fax: +33-4-72913401

C. Tilikete $\cdot$ N. Nighoghossian $\cdot$ A. Vighetto $\cdot$ D. Pélisson

Institut Fédératif des Neurosciences Lyon,

Hôpital Neuro-Cardiologique, Batiment B13, 59,

Bd Pinel, 69394 Lyon Cedex 03, France the patients. Indeed, the horizontal error of ipsilesional saccades in patients did not change significantly with the desired horizontal saccade size. In addition, when comparing saccades with similar intended direction, it was found that the directional error was inversely related to the vertical saccade amplitude. Thus we conclude that the cerebellum might be involved both in controlling the motor execution of saccades and in determining the visuo-spatial information about their goal.

Keywords Medullary lesion $\cdot$ Saccadic dysmetria Oculomotor cerebellum $\cdot$ Eye movements $\cdot$ Videooculography

Abbreviations cFN: Caudal fastigial nucleus

\section{Introduction}

Oculomotor and vestibular symptoms and signs are frequently encountered in Wallenberg's syndrome, an ischemic lesion of the lateral medulla. The lesion typically includes part of the vestibular nuclei and the inferior cerebellar peduncle (Carpenter and Cowie 1985; Morrow and Sharpe 1988). The majority of symptoms and signs, such as central vestibular nystagmus, vertigo, room tilt illusion, tilt of subjective visual vertical, skew deviation, ocular tilt reaction and angular displacement of posture, can be attributed to vestibular dysfunction (Hornsten 1974a, b; Milandre et al. 1995). However, the origin of other symptoms, such as saccadic lateropulsion, ocular lateropulsion or axial lateropulsion is much less certain since these symptoms may reflect either a vestibular imbalance or damage to other oculomotor and postural pathways in the inferior cerebellar peduncle (Leigh and Zee 1999).

Saccadic lateropulsion is characterized by an undershoot of contralaterally directed saccades, an overshoot of ipsilaterally directed saccades and ipsilateral deviation of vertical saccades (Kommerell and Hoyt 1973). Saccadic lateropulsion implies a saccadic step-size dysmetria, such as observed in patients with cerebellar lesions 
(Botzel et al. 1993). In animals with cerebellar lesion, stepsize saccadic dysmetria has been attributed to lesions of the oculomotor cerebellar vermis and/or of the caudal fastigial nucleus (cFN) that projects to brainstem oculomotor structures (Ritchie 1976; Optican and Robinson 1980; Vilis and Hore 1981; Robinson et al. 1993; Buttner et al. 1994; Goffart and Pelisson 1994; Ohtsuka et al. 1994; Straube et al. 1994; Goffart and Pelisson 1998; Takagi et al. 1998; Barash et al. 1999; Iwamoto and Yoshida 2002; Pelisson et al. 2003). The reciprocal effect of lesions affecting either the oculomotor cerebellar vermis or the $\mathrm{cFN}$ is consistent with the functional inhibition imposed by the former on the later. According to these data, it has been suggested that saccadic lateropulsion in Wallenberg syndrome and saccadic dysmetria due to cerebellar lesion have a common physio-pathological basis with the former resulting from an alteration of the olivo-cerebellar pathway (Straube et al. 1994). Further evidence supports this olivo-cerebellar pathway/oculomotor vermis/cFN involvement in saccadic lateropulsion. First, saccadic lateropulsion has been described in a patient with a lesion affecting the olivo-cerebellar pathway below its entrance in the inferior cerebellar peduncle (Tilikete et al. 2002). Second, animal studies have shown that a lesion to the inferior cerebellar peduncle interrupts climbing fibers, reducing complex spike activity in the ipsilateral vermis, leading to a reciprocal increase of simple spike activity and thus increased inhibition of the ipsilateral fastigial nucleus (Benedetti et al. 1983; Ohtsuka and Noda 1990; Ohtsuka and Noda 1991). Finally, contralateral saccadic lateropulsion which has been reported in patients with lesions of the $\mathrm{cFN}$ efferent pathways beyond the midline crossing point, i.e. "en route" to brainstem oculomotor structures, also supports this hypothesis (Ranalli and Sharpe 1986; Straube and Buttner 1994). In other words, lateral medullary infarction would produce a functional lesion of the ipsilateral FN.

The cerebellum has long been thought to be involved in the control of saccade amplitude. Since the high speed of saccades precludes any major role of visual feedback during the saccade, "on-line" saccadic control is considered to rely on internal efference-copy feedback guiding the eye to the target. The main class of models of the saccadic system assumes that the magnitude of a saccade is determined by an on-line comparison between desired eye displacement and an estimate of actual eye displacement (Robinson 1981). Some recent versions of these models suggest that the cerebellum plays an important part in this comparison between actual and desired eye displacement and that the cFN can help to stop the eyes when they have reached the desired position (Quaia et al. 1999; Thier et al. 2000). Animal data lead to two, non-exclusive, hypotheses to account for saccadic lateropulsion following cerebellar lesions. In the first hypothesis, saccadic inaccuracy results from an impairment in "motor" control processes, due to an altered internal online feedback signal of actual eye displacement (Kirkham et al. 1981; Vilis and Hore 1981; Quaia et al. 1999) and/or an altered acceleration/deceleration phase (Robinson et al. 1993; Takagi et al. 1998; Quaia et al. 1999). This hypothesis predicts a constant error in the gain of eye movements following $\mathrm{cFN}$ lesion, which would be revealed by an increase of saccade inaccuracy with the increasing size of desired saccade amplitude (Goffart and Pelisson 1994; Goffart and Pelisson 1998; Iwamoto and Yoshida 2002). It also predicts that saccadic directional error is constant for saccades of similar intended direction regardless of their intended amplitude. ${ }^{1}$ It also predicts that the error in the eye movement accumulates during the saccade. Therefore, if the lesion affects mostly the horizontal saccade generation, this would result in curved trajectories for oblique saccades. According to the second hypothesis, the saccadic inaccuracy would result from impairment in the "visual" control of the saccade, due to an altered encoding of visual target location or desired eye position. In this case, the error would result from an impairment of processes that encode the final goal of the saccade rather than control its execution online. This hypothesis predicts a "constant spatial error" in saccadic gaze shifts (Goffart and Pelisson 1998; Goffart et al. 1998), i.e. the direction and amplitude of the endpoint error is not dependent on the intended saccade size and direction. Since an error in encoding target location would take place prior to saccade onset, this hypothesis also predicts that the saccades should not show any abnormal curvature. In order to better understand the mechanisms of saccadic lateropulsion in Wallenberg syndrome and its relation to the cerebellar control of saccades, it is necessary to investigate the pattern of saccadic inaccuracy in two-dimensional space. To date, the few studies which have analyzed the pattern of saccadic dysmetria in Wallenberg syndrome have focused on horizontal saccades (Kirkham et al. 1981; Straube et al. 1994).

In the present study, we quantitatively analyzed the accuracy and the trajectory of oblique and vertical saccades in nine control subjects and five patients with saccadic lateropulsion due to Wallenberg syndrome. By comparing different saccade parameters between the two subject groups (Fig. 1), we sought to determine whether saccadic deficits result from a dysfunction of a motor or of visual control.

1. A motor guidance dysfunction would be suggested if the horizontal gain of eye movements is altered in a constant way. Therefore, we would expect that:

(a)The horizontal component of saccadic error increases with the size of horizontal target eccentricity.

(b)The saccadic directional error is constant for saccades of similar intended direction regardless of their intended amplitude.

\footnotetext{
${ }^{1}$ Indeed, saccade direction depends on the ratio of vertical saccade components divided by the horizontal saccade component $(\mathrm{V} / \mathrm{H})$. A change in horizontal gain by a factor $\mathrm{C}$ results in a saccade direction $\mathrm{V} /(\mathrm{C} \times \mathrm{H})=1 / \mathrm{C} \times \mathrm{V} / \mathrm{H}$. Thus the error in saccade direction due to the horizontal gain change $\mathrm{C}$ depends only on the ratio $\mathrm{V} / \mathrm{H}$ (i.e. the direction of the desired saccade) and does not change with saccade amplitude.
} 


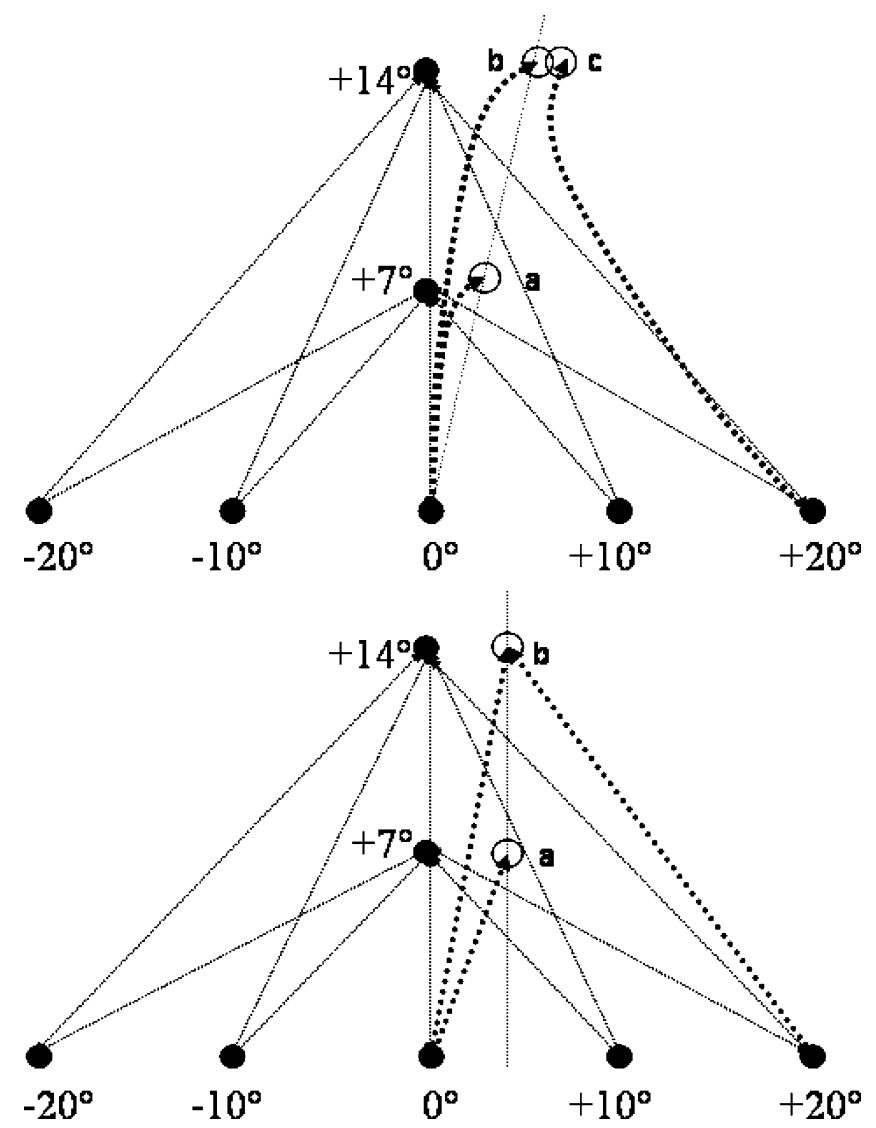

Fig. 1 Positions of LEDs (dots) and intended saccade directions (solid lines). Upward saccades start from the five different positions on the horizontal axis and are directed to the two different targets on the vertical axis (up $14^{\circ}$ and $u p 7^{\circ}$ saccades). Downward saccades start from the two different positions on the vertical axis $\left(\right.$ down $14^{\circ}$ and down $7^{\circ}$ saccades) and are directed to the five different targets on the horizontal axis. The top panel shows saccade trajectories (dotted lines) and final positions (open circles) for upward $7^{\circ}$ vertical saccades $(a)$, upward $14^{\circ}$ vertical saccades $(b)$ and for upward $14^{\circ}$ oblique saccade (c) predicted by the 'control of motor execution dysfunction' hypothesis. According to this hypothesis, the horizontal component of saccadic error increases with the size of both horizontal and vertical target eccentricity (saccade gain change) and the saccadic direction error is constant for saccades of similar intended direction regardless of their intended amplitude. Finally, according to this hypothesis, the error gradually accumulates during the saccade leading to abnormally curved (vertical and oblique) saccade trajectories. The bottom panel shows saccade trajectories and endpoints predicted by the "visual saccade target designation dysfunction' hypothesis (dotted lines and open circles). The horizontal component of saccadic error does not increase with target eccentricity. The saccadic direction error, however, is different for saccades of similar intended direction but different amplitude. The error is already present at the beginning of the saccade and saccade trajectory is straight

(c) The erroneous guidance of the ongoing saccade can lead to a curved saccade trajectory.

2. A visual guidance dysfunction would be suggested if the final position of the saccades starting from different initial positions is shifted horizontally in a constant way relative to the intended goal. Therefore, we would expect that:
(a)The horizontal component of saccadic error does not necessarily increase with the size of target eccentricity.

(b)The saccadic directional error is different for saccades of similar intended direction but different amplitudes.

(c) The erroneous guidance mechanisms occur before the execution of the saccade and thus do not predict curved saccade trajectories.

\section{Materials and methods}

Subjects

Five patients with saccadic lateropulsion related to a Wallenberg syndrome were included in this study (two females, three males; mean age: 64 years; age range: 49 78 years) (Table 1 ). The patients were recruited on the basis of their clinical neurological and oculomotor symptoms and on the location of their lesion as determined by magnetic resonance imaging (MRI). The ischemic lesion was unique and no patient presented an alteration of general medical state. Saccadic lateropulsion was ipsilateral to lesion in all patients, to the right in three patients and to the left in two patients. Patients were tested between 2.5 months and 30 years (No. 2) after the vascular event.

Nine control subjects were included in this study (five females, four males; mean age: 37 years; age range: $21-$ 54 years). They had no history of ophthalmological, vestibular or neurological disease.

Each subject gave informed consent in accordance with the requirements of the ethical committee.

\section{Clinical and radiological assessment}

The neuro-otological manifestations are presented in Table 2.

The clinical examination of eye movements included saccades, pursuit, high frequency vestibulo-ocular reflex, convergence, phoria, tropia and nystagmus in primary and lateral gaze (right, left, up, down). Saccadic lateropulsion was defined as a lateral bias affecting saccadic eye movements, which manifested itself as an ipsilesional deviation of vertical saccades with a hypometria of contralesional saccades and sometimes combined with a hypermetria of ipsilesional saccades. Clinical examination could show correcting centripetal saccades upon eye opening, indicating persistent eye deviation with eye closure. However, both clinical examination and video-oculographic recording of eye position did not show any eye deviation under visual fixation. An ipsilesional skew deviation was observed in two patients. Four patients initially experienced vertigo and three patients reported room tilt illusion. All patients showed spontaneous nystagmus in darkness. This nystagmus could be torsional or horizontal and in the latter case beating toward or away from the lesion. Nystagmus was absent under visual fixation at the time of recording. 
Table 1 Neurological manifestations of patients

\begin{tabular}{|c|c|c|c|c|c|c|c|c|c|c|}
\hline Patients & Age & Sex & Delay & Side & $\begin{array}{c}\text { IL Vest } \\
\text { sd }\end{array}$ & $\begin{array}{c}\text { IL Cb } \\
\text { sd }\end{array}$ & $\begin{array}{c}\text { IL } \\
\text { Horner }\end{array}$ & $\begin{array}{c}\text { IL Trig } \\
\text { deficit }\end{array}$ & $\begin{array}{c}\text { CL ST } \\
\text { deficit }\end{array}$ & $\begin{array}{c}\text { IL IX-X-XI } \\
\text { deficit }\end{array}$ \\
\hline$\# 1$ & 73 & F & $15 \mathrm{~m}$ & $\mathrm{R}$ & & & & & & \\
\hline$\# 2$ & 78 & $\mathrm{~F}$ & $30 \mathrm{y}$ & $\mathrm{R}$ & & & & & & \\
\hline$\# 3$ & 70 & $\mathrm{M}$ & $18 \mathrm{~m}$ & $\mathrm{R}$ & & & & & & \\
\hline$\# 4$ & 51 & $\mathrm{M}$ & $5 \mathrm{~m}$ & $\mathrm{~L}$ & & & & & & \\
\hline$\# 5$ & 49 & $\mathrm{M}$ & $\begin{array}{c}2,5 \\
\mathrm{~m}\end{array}$ & $\mathrm{~L}$ & & & & & & \\
\hline
\end{tabular}

The shaded boxes mean symptoms present at initial phase. Common manifestations of Wallenberg syndrome include vestibular syndrome, cerebellar syndrome, Horner sign, trigeminal deficit and velo-pharyngo-laryngo deficit on the ipsilesional side and body spino-thalamic deficit on the controlesionnal side

$V e s t$ Vestibular, $S d$ syndrome, IL ipsilesional, $C L$ contralesional, $C b$ cerebellar, Trig trigeminal, $S T$ spinothalamique, $m$ month, $y$ year, $R$ right, $L$ left, $F$ female, $M$ male

Table 2 Neuro-otological manifestations

\begin{tabular}{|c|c|c|c|c|c|c|}
\hline Patients & Side & Vertigo & Nystagmus & SVV & $\begin{array}{c}\text { Skew } \\
\text { Deviation }\end{array}$ & $\begin{array}{l}\text { Room tilt } \\
\text { illusion }\end{array}$ \\
\hline$\# 1$ & $\mathrm{R}$ & & R B & $\mathrm{R} 2^{\circ}$ & & $180^{\circ}$ \\
\hline \#2 & $\mathrm{R}$ & & $\begin{array}{c}\text { CCW } \\
\text { B\&GEN }\end{array}$ & $\begin{array}{c}\mathrm{R} \\
4,8^{\circ}\end{array}$ & & \\
\hline \#3 & $\mathrm{R}$ & & LB & $\begin{array}{c}\mathrm{R} \\
0,4^{\circ}\end{array}$ & $\mathrm{R} 4 \mathrm{~d}$ & $180^{\circ}$ \\
\hline$\# 4$ & $\mathrm{~L}$ & & LB & $L 1,3^{\circ}$ & & \\
\hline \#5 & $\mathrm{L}$ & & R B & L $3,5^{\circ}$ & L 2d & $90^{\circ}$ \\
\hline
\end{tabular}

The shaded boxes indicate symptoms present at initial phase. Skew deviation was specifically looked for and was defined as a vertical divergence of the eyes, independent of gaze direction. The side of skew deviation was defined as the side of the lower, i.e. hypotropic, eye regardless of which eye was actually at fault

$R$ Right, $L$ left, $C C W$ counterclockwise, $B$ beating, $G E N$ gaze evoked nystagmus, $S V V$ subjective visual vertical, $d$ dioptries

\section{Subjective visual vertical ( $S V V)$}

The visual perception of verticality was recorded for all subjects. Using a remote control, patients had to align a phosphorescent rod $\left(10^{\circ}\right.$ of visual angle long) with their subjective perception of verticality (ten trials) in total darkness. In the group of normal subjects, the mean SVV was $0.7^{\circ} \pm 1.2^{\circ}$. A significant tilt of SVV was considered (patients 2 and 5 ) when the value was outside the $\left(-1.7^{\circ}\right.$ to $3.1^{\circ}$ ) interval (mean \pm 2 standard deviation of the control group).

\section{Magnetic resonance imaging (Fig. 2)}

The location of the lesion was determined by MRI in the five patients. All patients showed a lateral medullary lesion without concomitant cerebellar infarction.

\section{Eye movement recording}

Horizontal and vertical movements of each eye were recorded with infrared video-oculography (SMI EyeLink ${ }^{\circledR}$ system, Germany). Subjects sat in a chair and were instructed to follow the visual targets with their eyes without moving their head. Any residual change of head position was monitored by a helmet mounted infra-red camera and was subsequently subtracted from the eye position data. Two other infrared cameras were mounted on the same $300 \mathrm{~g}$ helmet, one in front of each eye. Using contrast image detection, the horizontal and vertical positions of the center of the pupil of each eye were calculated on line.

Calibration parameters were established as follows: the subject was asked to look at successively illuminated LEDs, located on a panel placed $2 \mathrm{~m}$ away at $0^{\circ}, 20^{\circ}$ left, $20^{\circ}$ right on the horizontal line, $14^{\circ}$ up and $14^{\circ}$ down on the vertical line. Since patients with saccadic dysmetria often produced two or three saccades to reach the target, each eye position sample used for calibration was measured about $3 \mathrm{~s}$ following target presentation onset when stable ocular fixation of the target light was achieved. Following this calibration, saccades were elicited by asking the subject to gaze on illuminated LEDs at $0^{\circ}, 10^{\circ}$ and $20^{\circ}$ right and left along the horizontal meridian at the 


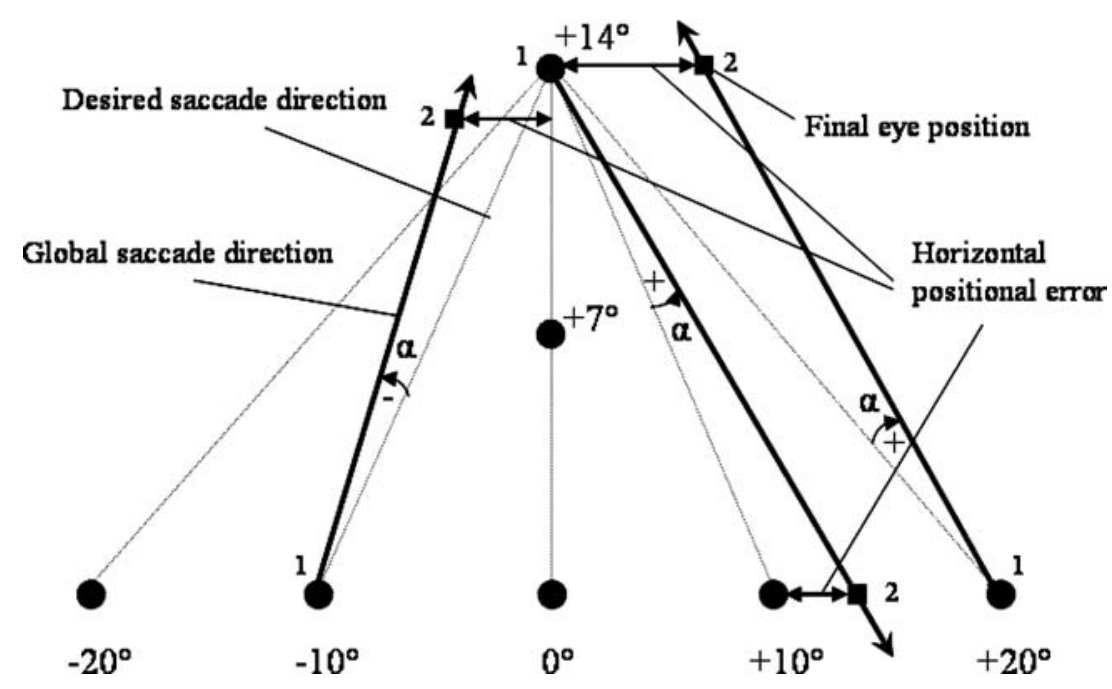

Fig. 2 Schematic illustration of horizontal positional error and global directional error. The horizontal positional error is defined as the horizontal separation between final eye position (black square labeled ' 2 ') and target position. The global saccade direction represented the orientation of the ideal vector linking initial (black circle labeled ' 1 ') and final eye positions. The desired saccade direction represented the orientation of the ideal vector linking the initial eye

position to the target position (thin dotted lines). The global directional error is given by $\alpha=$ global saccade direction - desired saccade direction. For both up and down saccades, positive values are assigned to rightward (controls) and ipsilesional (patients) errors and negative values are assigned to leftward (controls) and controlesional (patients) errors

subject's eye level or at $7^{\circ}$ and $14^{\circ}$ elevation along the vertical meridian. Targets were presented for $2 \mathrm{~s}$. By alternating the illumination of LEDs on the horizontal and vertical meridians, saccades with 20 different oblique or vertical directions were elicited (Fig. 1). The saccades were later grouped into upward saccades and downward saccades. Upward saccades started from the five different positions on the horizontal axis and were directed to the two different targets on the vertical axis (up $14^{\circ}$ and $u p 7^{\circ}$ saccades). Downward saccades started from the two different positions on the vertical axis $\left(\right.$ down $14^{\circ}$ and down $7^{\circ}$ saccades) and were directed to the five different targets on the horizontal axis. Thus, except saccades initiated from or to the central $\left(0^{\circ}, 0^{\circ}\right)$ position, all elicited saccades were oblique. We also grouped saccades according to the five horizontal retinal eccentricities: $-20^{\circ}$, $-10^{\circ}, 0^{\circ},+10^{\circ}$ and $+20^{\circ}$. In each session, two sets of 20 randomly elicited saccades were recorded. The session was repeated five times for each subject, with a short (1 min) break between each repetition. In total, 10 saccades for each of the 20 directions were elicited. All four calibrated signals, representing the horizontal and vertical position of each eye, were sampled by a second computer ( $500 \mathrm{~Hz}$ frequency), displayed and stored to disk for offline analysis (DataWave software, Berthoud, USA).

\section{Extraction of saccade parameters}

The gaze position and velocity data were processed by a PC software developed in our laboratory that performs waveforms filtering (FIR lowpass filter, $70-\mathrm{Hz}$ cutoff) and saccade detection on the basis of a velocity threshold criterion of $40 \%$ s. For each saccade, the following parameters were calculated for both the right and the left eye: saccade

latency, horizontal and vertical peak eye velocity, horizontal and vertical initial and final eye positions. These parameters were further processed and displayed by PCbased software (Statistica 6.1, Statsoft Inc. 1984-2003).

Subjects often made two or three saccades to reach the target. We focused our analysis on the initial (primary) saccade. Data from the two eyes were averaged to reflect cyclopean gaze position. We calculated the horizontal positional error as the horizontal separation between target position and final eye position (Fig. 2). The amplitude of the horizontal component of the saccades was calculated using initial and final eye positions. Three measures of saccade direction in 2-D space were performed. The global saccade direction was calculated using initial and final eye positions. The initial saccade direction was calculated using initial eye position and eye position at the point of maximal vectorial eye velocity. The desired saccade direction represented the orientation of the ideal vector linking the initial eye position to the target position. Based on these three saccade direction parameters, we calculated for each saccade the global directional error: "global direction-desired direction" ( $\alpha$ in Fig. 2), and the initial directional error: "initial direction - desired direction." The signs of all parameters (horizontal positional error, amplitude, initial and global directional errors) were adjusted for control subjects as positive for rightward errors and negative for leftward errors, and for patients as positive for ipsilesional errors and negative for contralesional errors.

\section{Statistical analysis}

Repeated measures ANOVAs were performed separately on horizontal positional error, horizontal amplitude, glo- 
bal directional error and the difference between initial and global directional errors, as obtained from the means of the ten-saccade repetitions. These three-way ANOVAs tested the effect of groups of subjects (control vs. patient; between subjects factor), the effect of saccade direction (up vs. down; within subject factor), the effect of vertical retinal eccentricity of the saccade target ( $7^{\circ}$ vs. $14^{\circ}$; within subject factor) and the effect of horizontal retinal eccentricity of the saccade target $\left(-20^{\circ},-10^{\circ}, 0^{\circ}\right.$, $10^{\circ}, 20^{\circ}$; within subject factor).

All statistics were performed by the STATISTICA software package (Statistica 6.1, Statsoft Inc. 19842003). A 95\% confidence interval was used to establish statistical significance.

\section{Results}

Aspects of the trajectories of the saccades

Figure 3 shows a two-dimensional representation of the trajectories of the $14^{\circ}$ upward and downward vertical and oblique saccades of the five patients. We plotted the trajectories of upward and downward saccades, between targets on the horizontal axis $\left(20^{\circ}\right.$ and $0^{\circ}$ to the right or left) and the $14^{\circ}$ vertical target, in the same figure in order to allow comparison between ipsilesional and contralesional saccades. Different elements can be pointed out from this figure. First, the saccades show a clear ipsilesional lateropulsion (to the right in patients 1,2 and 3, to the left in patients 4 and 5). Second, the saccades show a variable degree of curvature. With the exception of case 3, all patients exhibit directional errors at saccade onset. In two patients (cases 2 and 3 ) the curvature of the saccades tends to increase the final error in eye position $(B, C, G$, $\mathrm{H}$ ) whereas in two other patients (cases 4 and 5) the curvature tends to decrease the error (I, J). In some patients (cases 2 and 4), the ipsilesional saccades show a nearly linear trajectories (B, D, I) even though the contralesional saccades show a curved trajectory in the direction of the saccadic lateropulsion. In another patient (case 3), both the ipsilesional and the contralesional saccades show a curved trajectory in the direction of the saccadic lateropulsion $(\mathrm{C}, \mathrm{H})$. Finally, in some patients (cases 2 and 5), the trajectory of upward saccades differs from that of downward saccades (B, E, G, J).

In summary, the saccade trajectories tend to show different behaviors depending on the patient and the direction of the saccades. We could not demonstrate any relation with the degree of the lesion, neither the age of the lesion.

\section{Horizontal positional error}

The mean horizontal positional error for all saccades (oblique and vertical) was larger in the patient group $\left(1.8^{\circ} \pm 0.23^{\circ}\right)$ than in the control group $\left(0.05^{\circ} \pm 0.17^{\circ}\right)$ and deviated toward the lesion side (positive values)
$($ group effect $=$ control vs. patient; $F(1,12)=37$, $P<0.001)$. There was no significant difference in horizontal positional error of up versus down directed saccades (direction effect $=$ up vs. down; $F(1,13)=0.08$, $P>0.05)$. However, we did find a significantly different result in ipsilesional versus contralesional saccades in the patient group only (interaction between group and horizontal target eccentricity: $F(4,48)=4, P<0.01)$. As shown in Fig. 4a, in the control group, the horizontal error increased bilaterally with the size of horizontal target eccentricity (LSD tests, $P<0.05$ ). As shown in Fig. $4 \mathrm{~b}$, in the patient group the horizontal error increased with horizontal target eccentricity for contralesional saccades (LSD tests, $P<0.05$ ) but not for ipsilesional saccades (LSD tests, $P>0.05$ ). We found that the horizontal error did not change with the size of vertical target eccentricity (vertical target eccentricity effect $=+14^{\circ}$ vs. $\left.+7^{\circ} ; F(1,12)=3.74, P>0.05\right)$.

In summary, these analyses indicate that the horizontal positional error was significantly larger in patients as compared to controls, and that this difference (1) was not related to the vertical direction of the saccades and (2) was not related to vertical target eccentricity. Furthermore, ipsilesional and contralesional saccades in patients behaved differently. (1) The horizontal error of contralesional saccades increased with the size of horizontal target eccentricity. (2) For ipsilesional saccades the horizontal bias of the initial saccade endpoint did not change significantly as a function of horizontal target eccentricity.

Amplitude of the horizontal component of the saccades

The mean horizontal amplitude was not significantly different between the control group $\left(11.4^{\circ} \pm 0.21^{\circ}\right)$ and the patient group $\left(10.7^{\circ} \pm 0.26^{\circ}\right)$ (group effect; $F(1$, 13) $=3.9, P>0.05$ ). Only for the most contralesional saccades was the amplitude of the horizontal component of the saccade significantly shorter in the patient group than in the control group (LSD tests, $P<0.05$ ) (interaction between group and horizontal target eccentricity $F(4,48)=14.9, P<0.001)$. In both groups, the mean horizontal amplitude was not significantly different between the up and down direction (interaction between Group and direction; $F(1,12)=1.6, P>0.05)$, nor between the $7^{\circ}$ and $14^{\circ}$ vertical target eccentricities (VERECC, interaction between group and vertical target eccentricity; $F(1,12)=2.3, P>0.05)$.

In summary, we found in the patient group, an abnormal hypometria of horizontal component only for the most contralesional saccades. We did not find any hypermetria of the horizontal component of ipsilesional saccades in the patient group.

\section{Directional errors}

Directional errors were analyzed in two successive steps, providing first the global directional errors, and second the difference between initial and global directional errors. 
Fig. 3 Two-dimensional representation of individual saccade trajectories in the five patients. Left panels (a-e): upward saccades initiated from $20^{\circ}$ right, zero or $20^{\circ}$ left horizontal targets and directed toward the $14^{\circ}$ vertical target. Right panels $(\mathbf{f}-\mathbf{j})$ : downward saccades initiated from the $14^{\circ}$ vertical target and directed toward the $20^{\circ}$ right, zero and $20^{\circ}$ left horizontal targets. The saccades show a clear ipsilesional lateropulsion (to the right in cases 1,2 and 3, to the left in cases 4 and 5). Some saccades showed linear trajectory while other saccades showed curved trajectory. Ipsi for ipsilesional and Contra for contralesional saccades related to the direction of the lateropulsion

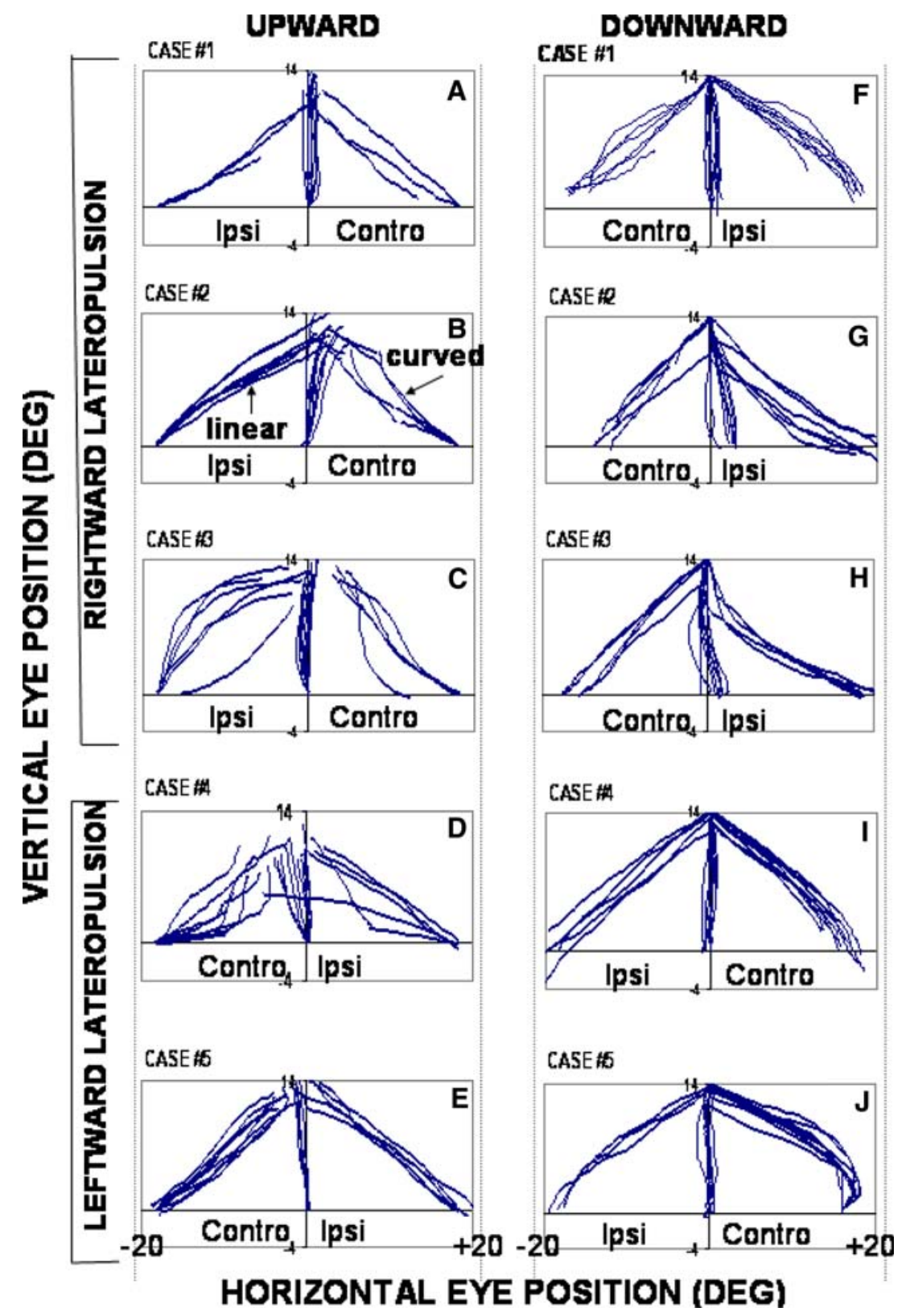

\section{Global directional errors}

The global directional errors were deviated toward the lesion in all saccades except for the most contralesional saccade. The mean global directional error for all saccades in the patient group $\left(+4.11^{\circ} \pm 0.56^{\circ}\right)$ was significantly different from the error of saccades in the control group $\left(+0.14^{\circ} \pm 0.42^{\circ}\right) \quad$ (group effect; $F(1,12)=32$, $P<0.001)$. Only the directional error for the most contralesional saccades in the patient group (Fig. 5b) failed to significantly differ from the error in the corresponding saccades in the control group (Fig. 5a) $(P>0.05$, LSD) (interaction between Group and horizontal target eccentricity; $F(4,48)=4.2, P<0.01)$. In both groups, there was no difference for saccades of different VERECC (group and vertical target eccentricity interaction;
$F(1,12)=1.3, P>0.05)$ nor for saccades with different direction, up versus down, (group and direction interaction; $F(1,12)=0.42, P>0.05)$.

To further evaluate whether saccade direction errors depend on saccade amplitude, we compared saccades of identical intended direction but of different amplitudes. We performed a repeated measure ANOVA on the three available pairs of saccades corresponding to intended oblique directions of $0^{\circ}$ (vertical: $0^{\circ} \mathrm{H}_{-} 7^{\circ} \mathrm{V}$ and $0^{\circ} \mathrm{H} \_14^{\circ} \mathrm{V}$ ), $+35^{\circ}$ (oblique contralesional: $+10^{\circ} \mathrm{H} 7^{\circ} \mathrm{V}$ and $+20^{\circ} \mathrm{H} 14^{\circ} \mathrm{V}$ ) or $-35^{\circ}$ (oblique ipsilesional: $-10^{\circ} \mathrm{H}$ $7^{\circ} \mathrm{V}$ and $-20^{\circ} \mathrm{H} 14^{\circ} \mathrm{V}$ ). The global directional error was larger for $7^{\circ}$ vertical eccentricity $\left(5.28^{\circ} \pm 1.7^{\circ}\right)$ than for $14^{\circ}$ vertical eccentricity $\left(3.24^{\circ} \pm 1.5^{\circ}\right)$ in the patient group (Fig. 6b), but was similar for $7^{\circ}\left(0.16^{\circ} \pm 1.3^{\circ}\right)$ and for $14^{\circ}\left(0.02^{\circ} \pm 1.1^{\circ}\right)$ in the control group (Fig. 6a) 
Fig. 4 Horizontal positional error (in degrees) in the control group (a) and the patient group (b) plotted as a function of the five horizontal target eccentricities (HORECC) and the two vertical target eccentricities (VERECC). In each group, we plot the horizontal error separately for $14^{\circ}$ vertical eccentricity (circle) and for $7^{\circ}$ vertical eccentricity (square). Error bars represent standard deviation. Positive values of horizontal positional error and HORECC are assigned to ipsilesional (patients) or rightward (controls) errors, while negative values are assigned to contralesional (patients) or leftward (controls) errors
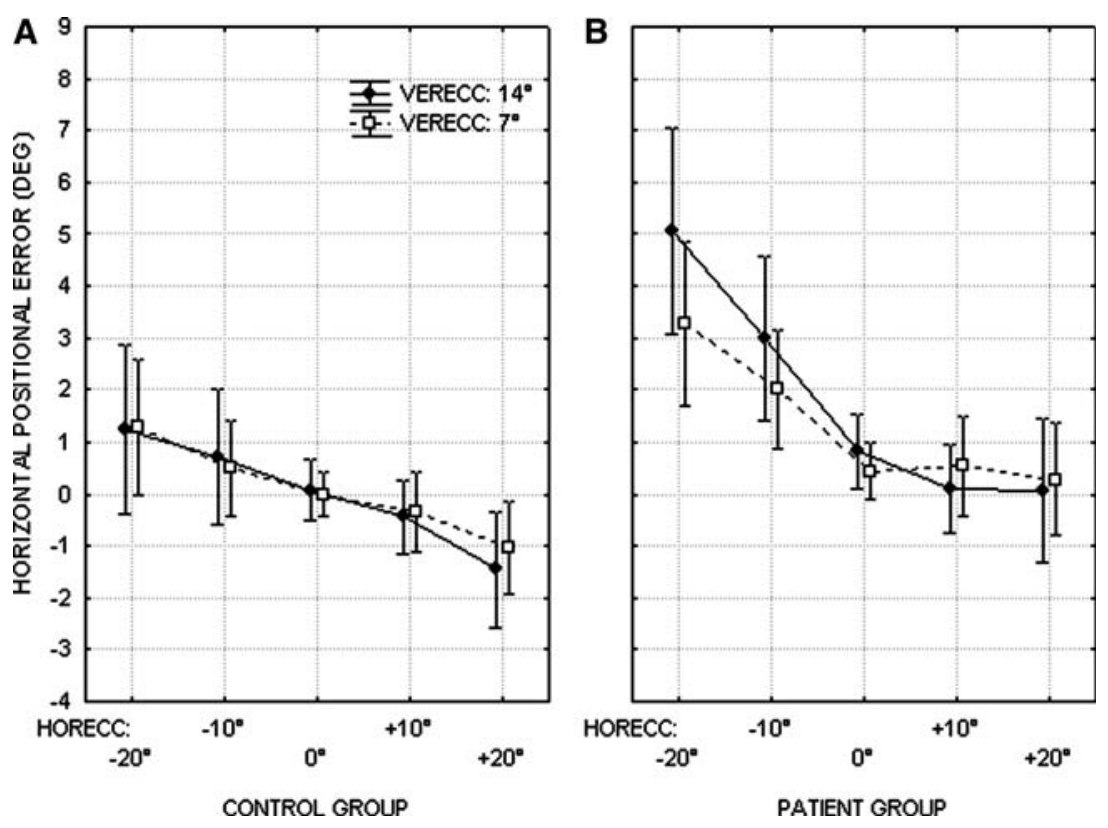

(group and vertical target eccentricity interaction; $F(1,12)=46, P<0.001)$.

In summary, the global directional error in the patient group was significantly larger than in the control group and was found to depend both on the horizontal and on the vertical retinal target eccentricity: (1) the global directional error was smaller for the most contralesional saccades; (2) the global directional error decreased with increasing vertical target eccentricity when testing saccades of similar intended direction along the vertical, contralesional oblique and ipsilesional oblique meridians.

\section{Difference between initial and global directional errors}

In order to evaluate the curvature of the saccades, we calculated the difference between the initial and the global directional errors. The difference between the initial and the global directional errors was close to zero in the control group $\left(-0.02^{\circ} \pm 0.08^{\circ}\right)$ (Fig. 5a) and negative in the patient group $\left(-3.5^{\circ} \pm 1.1^{\circ}\right)$ (Fig. $5 \mathrm{~b}$ ) (group effect; $F(1,12)=6.4, P<0.05)$. This means that the initial directional error was different from the global directional error in the patient group. As shown in Fig. 5b, the mean initial directional error was in opposite direction as compared to global directional errors for contralesional saccades, but was in the same direction and smaller for ipsilesional saccades (Fig. 5b). The difference between directional errors was larger at the $14^{\circ}$ vertical eccentricity $\left(-2.5^{\circ} \pm 2.97^{\circ}\right)$ than at $7^{\circ}\left(-1^{\circ} \pm 1.6^{\circ}\right)$ (vertical target eccentricity effect; $F(1,12)=6.1, P<0.05)$ (Fig. 5).

In summary, we found that the difference between initial and global directional error was negative in the patient group while this difference was absent in the control group, suggesting that for patients the trajectory curves ipsilesionally during the whole saccade execution.

\section{Discussion}

In the present study, we quantitatively analyzed different aspects of oblique and vertical saccades in patients with saccadic lateropulsion due to Wallenberg syndrome. The main goal of the study was to determine whether errors in the amplitude, direction and trajectory of saccades can be explained in terms of a dysfunction in the control of the execution of the movement (motor dysfunction) or in the designation of the movement target (visual dysfunction).

The first part of the discussion deals with the comparison of saccadic lateropulsion in our patients with previous reports on Wallenberg syndrome or cerebellar dysfunction. In the second part, we present evidence that saccadic errors in our patients can be accounted for by a prominent motor dysfunction. In the third part, we show in addition that some of our findings can be best interpreted in terms of a dysfunction in the visual control of saccades.

Comparison of saccadic lateropulsion in our patients with previous reports on Wallenberg syndrome or cerebellar lesion

The analysis of the horizontal component of the oblique saccades in our patients revealed that ipsilesional saccades were normometric and contralesional saccades were prominently hypometric. Previous reports in the literature suggest that patients with Wallenberg syndrome usually display both hypermetria of ipsilesional saccades and hypometria of contralesional saccades (Helmchen et al. 1994; Straube et al. 1994; Tilikete et al. 2002). The absence of ipsilesional hypermetria in our patients could be due to the different experimental design or related to the compensatory mechanisms that might have taken 
Fig. 5 Global (circle) and initial (square) directional error (in degrees) in the control group (a) and the patient group (b) plotted as a function of the five $\mathrm{HO}$ RECC and the two VERECC. Error bars represent standard deviation. Positive values of directional error and HORECC are assigned to ipsilesional (patients) or rightward (controls) errors, while negative values are assigned to contrale(controls) errors sional (patients) or leftward
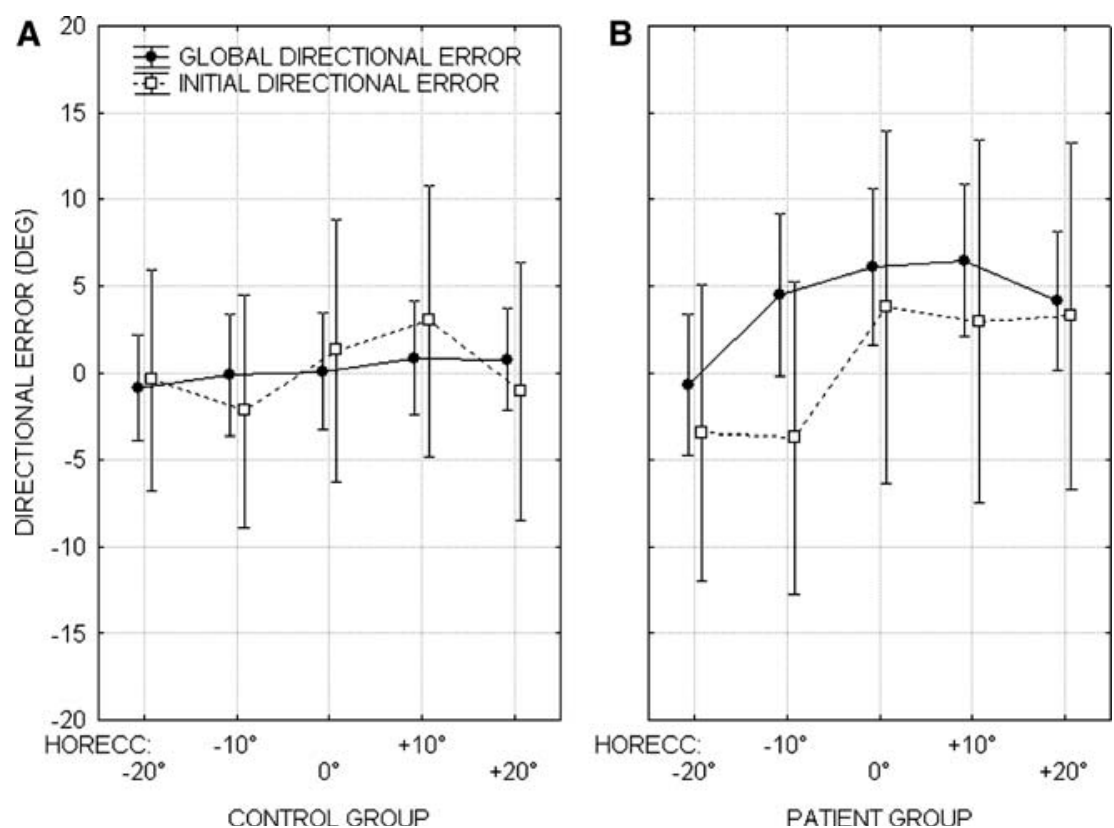

Fig. 6 Global directional error (in degrees) in the control group (a) and the patient group (b) plotted as a function of three pairs of similar intended directions (INDIR: $-35^{\circ}, 0^{\circ},+35^{\circ}$ ) and the two vertical target eccentricities (VERECC: $7^{\circ}$ and $14^{\circ}$ ) (same format as in Fig. 5). Error bars represent standard deviation

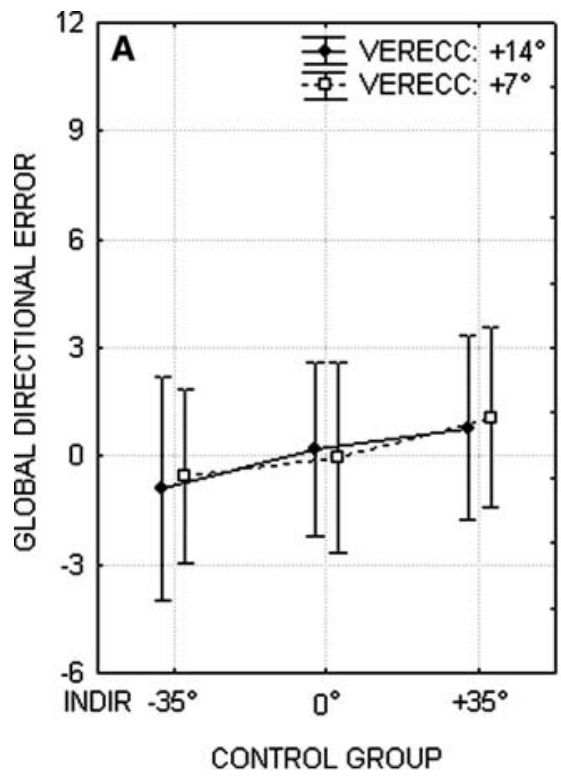

place at the time of recording, i.e. several months after the stroke (Kirkham et al. 1981; Straube et al. 1994). Furthermore, in at least one of our patients, the initial direction of the oblique or vertical saccades was deflected contralaterally relative to the saccadic lateropulsion, suggesting some other kind of compensatory strategy. In this study, we were interested by the long-term persistence of saccadic lateropulsion. Even if compensation would have minimized the ocular phenomenon, it should not change the origin of errors in saccades. Indeed, the global direction of the vertical and oblique ipsilateral saccades made by our patients was deflected to the ipsilesional side, revealing a persistent lateropulsion of these saccades (Meyer et al. 1980; Kirkham et al. 1981; Helmchen et al.
1994; Solomon et al. 1995). In addition, as discussed in the following paragraph, the relationship between saccadic horizontal error and target eccentricity disclosed in patients differed from that in control subjects.

Experimental evidence for a similarity between saccadic lateropulsion in Wallenberg syndrome and saccadic dysmetria with cerebellar lesions has been provided in the introduction. Our study adds a new element by showing in humans a qualitative difference between ipsilesional and contralesional saccades, in agreement with the difference observed in head-free gaze shifts in cats with cFN lesion (see Fig. 8 in Goffart and Pelisson 1998). Both in cats and in our patients, ipsilesional saccades exhibited a constant horizontal error while contrale- 
sional saccades showed an error that increased with the size of the saccade. Such a difference of behavior between ipsilesional and contralesional saccades was not reported in primate models of unilateral cerebellar lesions (Robinson et al. 1993; Iwamoto and Yoshida 2002; Goffart et al. 2004).

Our results are therefore consistent with the hypothesis that saccadic lateropulsion in Wallenberg syndrome is the consequence of a dysfunction of the cerebellar control of saccades (Helmchen et al. 1994; Straube et al. 1994). Whether saccadic lateropulsion is the result of an alteration of, respectively, the motor signal of eye position or the visual signal of target position in space (or desired eye position) needed by the cerebellum to control visually guided saccades will now be addressed.

Saccadic lateropulsion as the result of a motor control dysfunction?

Some of the saccadic behavior observed in our patients can most easily be interpreted in terms of a dysfunction in the control of the motor execution of saccades.

First, we observed that the horizontal error of the primary contralesional saccades increases with horizontal target eccentricity. Second, the trajectory curves ipsilesionally during the whole saccade execution in the patients, whereas initial and global directions were not different in the control group. This result suggests that directional errors could gradually develop during the ongoing saccade. Indeed, some of the patients showed curvature in their saccade trajectories. Altogether, these results suggest that the saccade gain is altered in a constant way. This could involve the gain of the efferencecopy feedback signal for tracking current eye position. This conclusion is in accordance with that of previous studies in Wallenberg syndrome and in primate and cat with $\mathrm{cFN}$ inactivation showing a constant hypometric gain of contralesional saccades (Kirkham et al. 1981; Robinson et al. 1993; Goffart and Pelisson 1998; Iwamoto and Yoshida 2002). It has been suggested that cFN neurons control the motor aspect of contralesional saccades by excitation of burst neurons and inhibition of pause neurons of the contralateral reticular formation (Noda et al. 1990; Robinson et al. 2002; Scudder and McGee 2003).

Saccadic lateropulsion as the result of a visual dysfunction?

Some of our results cannot readily be explained in terms of a motor dysfunction. These results are better explained by assuming a constant bias in the signal specifying the target position in space.

Specifically, and contrary to the increase observed for contralesional saccades, we found that the horizontal error of ipsilesional saccades remained constant as a function of horizontal target eccentricity. In addition, the directional error of saccades of the same intended direction was inversely related with their vertical amplitude, suggesting a constant spatial error. Finally, some patients did not show any significant curvature in their saccade trajectories. Taken together, these results suggest that the processes that specify the goal of the saccades might be impaired in patients with saccadic lateropulsion, resulting in a horizontal shift of desired eye position. This conclusion is compatible with studies performed in cats (Goffart and Pelisson 1994; Goffart and Pelisson 1998) but not in a recent study performed in the monkey (Goffart et al. 2004). Note, however, that in humans with saccadic lateropulsion, saccades made in total darkness and memory guided saccades are not affected or less affected than saccades toward visual targets (Kirkham et al. 1981). These results putatively suggest an impairment in computing the spatial direction in which to move the eyes to reach a visible target.

We suggest that the cerebellum might help to compute the direction or desired endpoint of saccadic eye movements triggered by a visual target. A possible substrate for this cerebellar contribution are the bilateral projections of the cFN toward both superior colliculi (see Pelisson et al. 2003 for references). The deep layers of the superior colliculus play a key role in encoding saccade metrics and in keeping memory target-related information until completion of the saccadic response (Sparks 1999; Bergeron and Guitton 2001). Therefore, modification of the cFN activity through lesion of the olivo-cerebellar pathway, such as in Wallenberg syndrome, or through direct inactivation in animals, could alter the collicular mechanisms that keep accurate information about the saccade goal, resulting in a bias of ipsilesional saccades and possibly some components of the contralesional saccade deficits.

\section{Conclusions}

Saccadic lateropulsion is observed in Wallenberg syndrome due to a lesion affecting the olivo-cerebellar pathway. The similarity between saccadic lateropulsion in patients and the saccadic dysmetria observed in experimental inactivation of the $\mathrm{cFN}$ suggests a common pathophysiology involving the oculomotor cerebellum. This cerebellar oculomotor network has for a long time been considered to play a predominant role in on line motor control of saccades. Our observations of patients with saccadic lateropulsion support this view and further suggest that the cerebellum might also be involved in processing the visuo-spatial location of the saccade goal prior to movement initiation. These findings suggest that other visuo-motor biases observed in Wallenberg syndrome, such as the ipsilateral shift of the postural axis, may also result from alterations in cerebellar-mediated mechanisms encoding visual signals.

Acknowledgments This work was supported by "Projet de Recherche Clinique des Hospices Civils de Lyon" Grant n HCL/P/99 201. We thank Mrs Chloe Hutton for English corrections of the manuscript. 


\section{References}

Barash S, Melikyan A, Sivakov A, Zhang M, Glickstein M, Thier P (1999) Saccadic dysmetria and adaptation after lesions of the cerebellar cortex. J Neurosci 19:10931-10939

Benedetti F, Montarolo PG, Strata P, Tempia F (1983) Inferior olive inactivation decreases the excitability of the intracerebellar and lateral vestibular nuclei in the rat. J Physiol 340:195-208

Bergeron A, Guitton D (2001) The superior colliculus and its control of fixation behavior via projections to brainstem omnipause neurons. Prog Brain Res 134:97-107

Botzel K, Rottach K, Buttner U (1993) Normal and pathological saccadic dysmetria. Brain 116(Pt 2):337-353

Buttner U, Straube A, Spuler A (1994) Saccadic dysmetria and "intact" smooth pursuit eye movements after bilateral deep cerebellar nuclei lesions. J Neurol Neurosurg Psychiatry 57:832-834

Carpenter MB, Cowie RJ (1985) Connections and oculomotor projections of the superior vestibular nucleus and cell group ' $y$ '. Brain Res 336:265-287

Goffart L, Chen LL, Sparks DL (2004) Deficits in saccades and fixation during muscimol inactivation of the caudal fastigial nucleus in the rhesus monkey. J Neurophysiol 92:3351-3367

Goffart L, Pelisson D (1994) Cerebellar contribution to the spatial encoding of orienting gaze shifts in the head-free cat. J Neurophysiol 72:2547-2550

Goffart L, Pelisson D (1998) Orienting gaze shifts during muscimol inactivation of caudal fastigial nucleus in the cat. I. Gaze dysmetria. J Neurophysiol 79:1942-1958

Goffart L, Pelisson D, Guillaume A (1998) Orienting gaze shifts during muscimol inactivation of caudal fastigial nucleus in the cat. II. Dynamics and eye-head coupling. J Neurophysiol 79:19591976

Helmchen C, Straube A, Buttner U (1994) Saccadic lateropulsion in Wallenberg's syndrome may be caused by a functional lesion of the fastigial nucleus. J Neurol 241:421-426

Hornsten G (1974a) Wallenberg's syndrome. I. General symptomatology, with special reference to visual disturbances and imbalance. Acta Neurol Scand 50:434-446

Hornsten G (1974b) Wallenberg's syndrome. II. Oculomotor and oculostatic disturbances. Acta Neurol Scand 50:447-468

Iwamoto Y, Yoshida K (2002) Saccadic dysmetria following inactivation of the primate fastigial oculomotor region. Neurosci Lett 325:211-215

Kirkham TH, Guitton D, Gans M (1981) Task dependent variations of ocular lateropulsion in Wallenberg's syndrome. Can J Neurol Sci 8:21-26

Kommerell G, Hoyt WF (1973) Lateropulsion of saccadic eye movements. Electro-oculographic studies in a patient with Wallenberg's syndrome. Arch Neurol 28:313-318

Leigh RJ, Zee DS (1999) The neurology of eye movements. F.A. Davis Company, Philadelphia

Meyer KT, Baloh RW, Krohel GB, Hepler RS (1980) Ocular lateropulsion. A sign of lateral medullary disease. Arch Ophthalmol 98:1614-1616

Milandre L, Lucchini P, Khalil R (1995) Lateral bulbar infarctions. Distribution, etiology and prognosis in 40 cases diagnosed by MRI. Rev Neurol (Paris) 151:714-721

Morrow MJ, Sharpe JA (1988) Torsional nystagmus in the lateral medullary syndrome. Ann Neurol 24:390-398
Noda H, Sugita S, Ikeda Y (1990) Afferent and efferent connections of the oculomotor region of the fastigial nucleus in the macaque monkey. J Comp Neurol 302:330-348

Ohtsuka K, Noda H (1990) Direction-selective saccadic-burst neurons in the fastigial oculomotor region of the macaque. Exp Brain Res 81:659-662

Ohtsuka K, Noda H (1991) The effect of microstimulation of the oculomotor vermis on discharges of fastigial neurons and visually-directed saccades in macaques. Neurosci Res 10:290-295

Ohtsuka K, Sato H, Noda H (1994) Saccadic burst neurons in the fastigial nucleus are not involved in compensating for orbital nonlinearities. J Neurophysiol 71:1976-1980

Optican LM, Robinson DA (1980) Cerebellar-dependent adaptive control of primate saccadic system. J Neurophysiol 44:10581076

Pelisson D, Goffart L, Guillaume A (2003) Control of saccadic eye movements and combined eye/head shifts by the medio-posterior cerebellum. Elsevier, Amsterdam

Quaia C, Lefevre P, Optican LM (1999) Model of the control of saccades by superior colliculus and cerebellum. J Neurophysiol 82:999-1018

Ranalli PJ, Sharpe JA (1986) Contrapulsion of saccades and ipsilateral ataxia: a unilateral disorder of the rostral cerebellum. Ann Neurol 20:311-316

Ritchie L (1976) Effects of cerebellar lesions on saccadic eye movements. J Neurophysiol 39:1246-1256

Robinson DA (1981) The use of control systems analysis in the neurophysiology of eye movements. Annu Rev Neurosci 4:463-503

Robinson FR, Fuchs AF, Noto CT (2002) Cerebellar influences on saccade plasticity. Ann N Y Acad Sci 956:155-163

Robinson FR, Straube A, Fuchs AF (1993) Role of the caudal fastigial nucleus in saccade generation. II. Effects of muscimol inactivation. J Neurophysiol 70:1741-1758

Scudder CA, McGee DM (2003) Adaptive modification of saccade size produces correlated changes in the discharges of fastigial nucleus neurons. J Neurophysiol 90:1011-1026

Solomon D, Galetta SL, Liu GT (1995) Possible mechanisms for horizontal gaze deviation and lateropulsion in the lateral medullary syndrome. J Neuroophthalmol 15:26-30

Sparks DL (1999) Conceptual issues related to the role of the superior colliculus in the control of gaze. Curr Opin Neurobiol 9:698707

Straube A, Buttner U (1994) Pathophysiology of saccadic contrapulsion in unilateral rostral cerebellar lesions. Neuroophthalmology 14:3-7

Straube A, Helmchen C, Robinson F, Fuchs A, Buttner U (1994) Saccadic dysmetria is similar in patients with a lateral medullary lesion and in monkeys with a lesion of the deep cerebellar nucleus. J Vestib Res 4:327-333

Takagi M, Zee DS, Tamargo RJ (1998) Effects of lesions of the oculomotor vermis on eye movements in primate: saccades. J Neurophysiol 80:1911-1931

Thier P, Dicke PW, Haas R, Barash S (2000) Encoding of movement time by populations of cerebellar Purkinje cells. Nature 405:7276

Tilikete C, Hermier M, Pelisson D, Vighetto A (2002) Saccadic lateropulsion and upbeat nystagmus: disorders of caudal medulla. Ann Neurol 52:658-662

Vilis T, Hore J (1981) Characteristics of saccadic dysmetria in monkeys during reversible lesions of medial cerebellar nuclei. J Neurophysiol 46:828-838 